\title{
Exploiting Contextual Handover Information for Versatile Services in NGN Environments
}

\author{
Edson D. S. Moreira \\ University of São Paulo \\ Department of Computer Systems \\ edson@icmc.usp.br \\ Jon Crowcroft \\ University of Cambridge \\ Computer Laboratory \\ jon.crfowcroft@cl.cam.ac.uk \\ Glenford E. Mapp \\ Middlesex University \\ School of Computer Science \\ g.map@mdx.ac.uk
}

\begin{abstract}
Users in ubiquitous and pervasive computing environments will be much more empowered in ways to access and to control their navigation. Handover, the vital event in which a user changes the attachment point in a Next Generation Network $(N G N)$, is an important occasion and the conditions and environment in which it is executed can offer relevant information for businesses. This paper describes the capabilities of a platform which intends to exploit contextual handover information offering a rich environment that can be used by access and content providers for building innovative context-aware multi-provided services. Based on ontologies, the technique not only eases the building of versatile services but also provides a comprehensive source of information both for enriching user navigation in the network as well as for the improvement of the provider's relationship with their customers.
\end{abstract}

\section{Moving, sensing and switching}

Future computing will be based upon the idea that the users are highly mobile, their devices are ubiquitously instrumented to sense the surroundings, and will be continuously interacting with local and remote environments. Sensors will look for signs of locally emanated events, objects, people and services of interest to the user. Users will also use communicating channels to interact with remote

\author{
David N. Cottingham \\ University of Cambridge \\ Computer Laboratorry \\ david.cottingham@cam.ac.uk \\ Pan Hui \\ University of Cambridge \\ Computer Laboratory \\ pan.hui@cl.cam.ac.uk \\ Renata M. P. Vanni \\ University of Sao Paulo \\ Department of Computer Systems \\ rporto@icmc.usp.br
}

environments, looking for information on events, objects, people and services elsewhere. The mobile user, be it inside a private car or on public transportation, or even inside a public place or at home, will be inserted in contexts which are rich in information.

Concurrently to the actions associated with finding the signals for choosing the point of connection to the network, the user, through their devices, will be sensing other environmental parameters, as seen in the case of the Vehicular Ad Hoc Networks (VANET) [2], Body Sensor Networks (BSN) [20] and Personal Area Networks (PAN) [13]. Meteorological sensors (for temperature, humidity, barometric pressure) will help to establish the environmental conditions in which the user is embedded. Positioning and the speed of the user are also very important parameters in the process as well as other space-based information being given by accelerometers, magnetometers, etc. as prototyped in the Sentient Vehicles project [15]. It seems to us that if all this information is federated at the moment of network handover, rich correlations could be made, creating the conditions for building innovative services.

The mobile user will be constantly switching the point of attachment, and sometimes the technology, of connection to the network. Corporate WLANs, cellular networks (GSM or UMTS), public wireless networks, personal networks, and Vehicular Ad Hoc Networks are some possibilities for connection. Innovative modes of interaction and organization 
between a community of mobile users are envisaged and indeed start to materialize (mesh community wireless, pocket switched networks, sensor networks) creating complex scenarios which greatly differ from the usual user-provider relationships.

Each time a user device changes the point of attachment to the network a handover occurs [18]. Each handover can be a complex process, as it can depend on and indeed influences many variables: access provider (and their policies on QoS, privacy, security, pricing), wireless technologies (802.11, UMTS, WiMax), ecosystem in which the user is embedded (a tunnel, a traffic jam, darker or lighter environments, noisy places), etc. Handovers are essential mechanisms for the functioning of the cellular networks and users of the system barely notice them. The handovers in cellular technologies are mobile-assisted, but the decision to perform a handover is network-controlled. On the other hand, Mobile IP has been designed for IP networks where the management of the network is distributed, with end users having as much control over the traffic as do the core entities in the network.

A handover can occur for several reasons. It can be imperative (when the user drives away from a hotspot and the only option is to switch to a cellular network to keep the connection); by convenience (e.g, when the user enters a hospital and wants to use the services provided by its internal network: maps, search for a doctor, making appointments, etc); by incentives (when the user is offered some kind of benefit to attach to a specific network); by preference (when a user decides to switch the attachment point based on a previous good experience: pricing, reliability, security, privacy, etc); by opportunism (when the user gets into a community network for which they have a payload to deliver or to pick up). Whatever the reason, the contextual information around the moment the handover is made can be used for business purposes.

This paper deals with the possibilities that can be exploited by service providers (be it for access or for content) to build value added services through a good strategy of using context information when handovers occur. The proposal of structuring the relevant information into an ontology, besides creating the common agreed terminology which will facilitate the integration of services, gives them the semantics which would permit the search, the combination and proper correlation of capabilities for the services they will offer.

\section{Business in the tower of Babel: Using ontologies to facilitate information sharing for business}

Several entities have an interest in the information available at the point of handover. Each one of these entities could belong to a different area of business and present different nomenclature on the parameters concerning their services. However, all of them will need to cooperate with each other in order to obtain the maximum benefit. The ontology tries to create a common vocabulary of terms to support the services. Such a vocabulary can provide a way to normalize concepts in a new area of business which does not yet have a good set of commonly defined terms. For instance, naming types of interfaces, parameters for quality of service, terms related to security or privacy policies must be agreed between the providers. The sharing of the information by the various entities involved in the provision of a service has several impacts:

- From the user's perspective, they can choose the providers based upon several criteria: best response, best matching to his/her requirements, economy (use a home user-owned WLAN, during a traffic jam). They can use contextual information to adapt the user device profile of usage: power management (CPU, memory, display, network interface), streaming control (proximity to known blind spots such as tunnels), optimization of content delivery to a new profile.

- From the access provider's perspective, it might be interesting to grab information to improve the long term relationships with the user such as his/her choices of connection/disconnection times and locales. It may collect information about the handovers of its users and relate them with positioning information, indicating, for instance, blind spots, traffic jams, weather conditions, etc. The access provider may also correlate information about user's routes and timing of accesses. In general, it can understand the criteria by which the user chooses the access providers.

- From the content provider's perspective, they may adapt the delivery of media to specific devices, location, timing, type of user, etc. They can explore the contextual information to add value to the content (advertisement, linking to 3rd party products, etc). As the user has control over the mobility aspects, the access provider can focus on providing better, environmentally oriented and more varied services. Providers can also provide brokerage services based on the common information available. 


\section{How ontologies can help to transform handover information into business opportunities}

In contrast to the usual database information structure, which is focused on an application, the ontology structure is focused on a domain. Figure 1 shows the overall Service Oriented Handover (SOHand) Management System architecture that exploits the idea: an abstract platform (an interface) to support the delivery of advanced network services. The basis of the system is a good, versatile, information structure, an ontology, which could be shared by providers and users, a kind of distributed Register Profile (RP), in the telephone industry or Mobile Information Device Profile (MIDP) in the Mobile web services proposals. At the user level a SIM-IP, the internet version of the Subscriber Identity Module could serve as the repository and manager of local information. The ontology would be the unifying technology, providing a common understanding of the terms and relationships which could be jointly maintained by the providers. The Ontology Service, stored in distributed servers at the user, access providers and content providers, will provide data about the user, application and environment contexts.

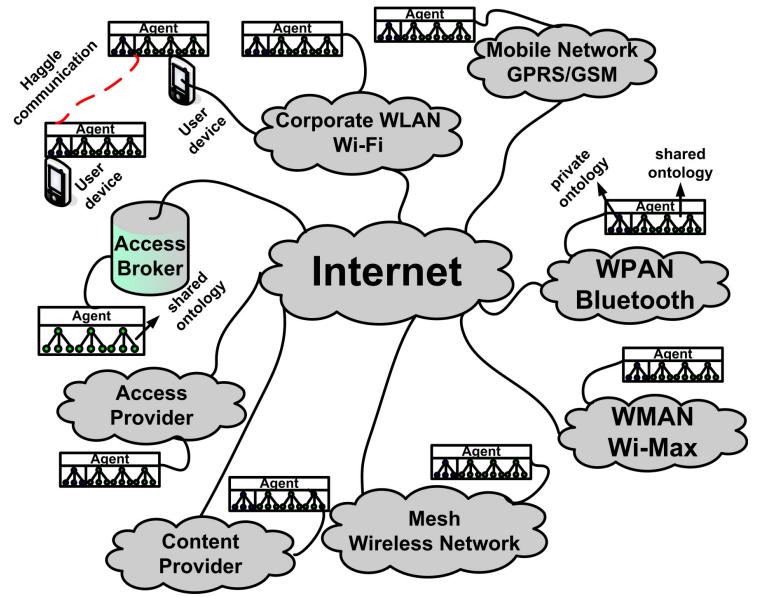

Figure 1. The SOHand Architecture

The user side of the applications would contain agents able to access the environmental sensors, the provider's information and the SIM-IP so the user could benefit from the combination and correlation of the information to enrich their experience. Multihomed devices equipped with several interfaces could use the ontologies to assist roaming, being able to dynamically influence the handover process (e.g. network selection).

An empowering role is given to the users with their capacity of controlling the interaction with the network and having greater choice, both in terms of connectivity and services. On handover events, the session entity will take care of the change of the policies, making the proper mappings and adjustments. This architecture is very similar to others proposed for multi-provider wireless services such as the one originating in the MMQoS project, but has some key differences. Besides the usual connection to the internet via a provider (what is commonly called infra-structured access), in this model users can also make occasional, opportunistic, communications with other devices in the vicinity (what is commonly known as ad hoc access). This is illustrated in Figure 1, where two user devices make a direct connection between themselves. The other difference is the user-centered approach of management, instead of the provider-centered one. The ontology classes and their relationships are shown in Figure 2.

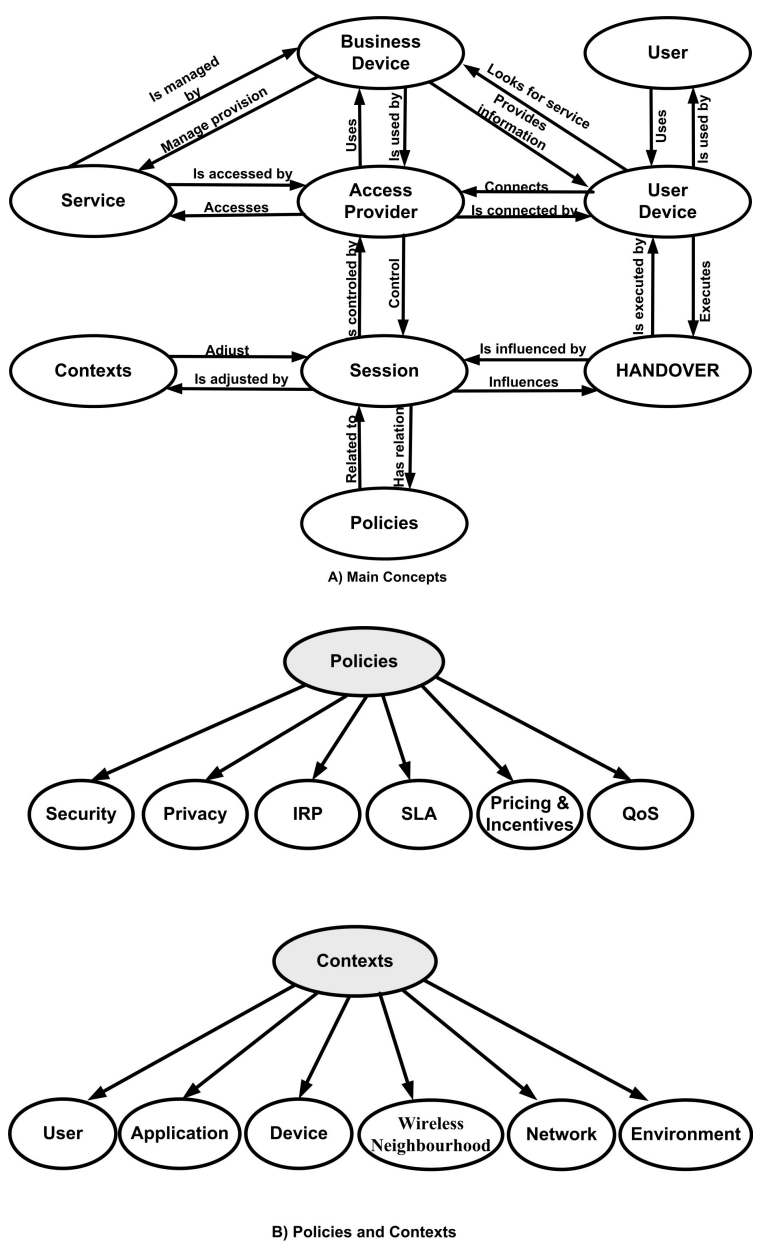

Figure 2. Main Concepts and Relationships

In SOHand a service is a facility (directory information, a transaction, a message exchange, a video stream, a voice channel, a game application) which a user or a content provider offers, during a 
session, to a user through an access provider or by a direct connection. One entity can offer both access and content at the same time. In the course of enjoying a service, the user seamlessly roams through a net of access providers, be they traditional ones or informal organizations like the mesh wireless community networks, and the clouds of other users' radio antennas, switching from one to another in procedures which are called handovers. The service is managed by the business device, which acts as a broker between the user and the provider, if this exists. Context information such as that related to the user, the environment, the network, etc, gathered by positioning, climate, radio and other sensors and from the user device or by any other related service, can be used by the session to frame and map Security, Privacy, QoS, Billing, SLA and other policies.

The Business Device is the entity which will help the user with the business side of the service, when required (Authentication, billing and measurements of the quality-of-experience of the user). The Access Provider is a class with several sub-classes, each one of them in charge of defining a different kind of provision. It could be a traditional, cellular or hotspot based service or it could be a service regulated by a wireless mesh community or, still, it could be an occasional, opportunistic encounter of two mobile devices needing to exchange information, in which case the Access Provider is null.

\section{Sentient vehicles and pocket switched networks}

This is an ongoing project which was started in order to understand the domain, the definition of terms and concepts involved in the area and devise business models which would be used to instantiate the system. We are particularly mindful of following established or good emerging standards in the implementation of this project. We are trying, as much as possible, to follow the SOUPA (Standard Ontology for Ubiquitous and Pervasive Applications) framework [4]. This is an interesting model which divides the system into core ontologies and extension ontologies. SOUPA Core defines a wide range of concepts and relationships that are of general use for different ubiquitous applications. SOUPA Extension defines vocabularies for specific types of applications. We understand that, for the SOHand platform, the core ontology will take care of the common vocabulary which will be shared by all the entities belonging to the environment, such as the ones shown in Figure 1. They will be understood by all the entities involved in the provision of a service. Some of the extensions will be designed by the providers in order to create their own private vocabularies, many of them will not be available to the other (most of the times competing) entities.

Our test scenario is built upon the integration of 2 projects being carried at the Computer Laboratory at the University of Cambridge. We will next describe these projects and show how SOHand can exploit both the richness of the contextual information provided by the Sentient Van and the possibilities open using the Haggle opportunistic networking architecture.

\subsection{Sentient Vehicles}

Most people spend a reasonable amount of their daily time on the road. Whereas this is sometimes inevitable, one wants to maximize the use of this time by reading, getting informed and better coping with the other demands of modern life. The Intelligent Transportation Systems (ITS) community advocates the use of computing information resources to boost the transportation system's efficiency, better the environment and increase the well being of the people inside the vehicles. Information from the sensors in the car, used for engine management, cruise control or safety systems such as ABS braking, can be gathered and made available through VANETs. The combination of this information with data collected by environmental sensors such as meteorological and positioning technologies forms the basis of the Sentient Vehicles project [15].

Data from the various sensors in the vehicle are logged to hard disk, and is also available for transmission back to base and to other vehicles over wireless networks. This means that vehicles can act as highly mobile sensing platforms, which can share and combine sensor information from each other, as well as obtain data from the Internet. Using this processed sensor data, network operators will be able to gain key insights into both the behaviour of their users under particular conditions, and into the performance of their networks at particular geographical locations, rather than in general areas. Detailed and temporally accurate coverage maps of wireless technologies will be possible using this sensing infrastructure, which will then allow user equipment to pro-actively predict when handovers should occur, perhaps due to network blackspots, or what route a user should take in order to achieve fewest handovers [21]. The sharing of this type of information between user and provider, and user to user necessitates a common ontology that allows the portability of this type of knowledge. 
Table 1. Some ways to keep services sentient to the surrounding conditions

\begin{tabular}{|c|c|c|c|}
\hline Service & Parameters Involved & $\begin{array}{l}\text { Policies Involved } \\
\text { (see Fig. 2) }\end{array}$ & $\begin{array}{l}\text { Contexts involved } \\
\text { (see Fig. 2) }\end{array}$ \\
\hline \multirow{6}{*}{$\begin{array}{l}\text { Video } \\
\text { Broadcasting }\end{array}$} & Type of application & SLA & Application \\
\hline & Demand of QoS & QoS & Service \\
\hline & Demand for privacy & Privacy & Application, Environment \\
\hline & Demand for security & Security & Application, Environment \\
\hline & Time to Next Handover & & Network \\
\hline & $\begin{array}{l}\text { Connection options in the } \\
\text { neighbourhood }\end{array}$ & SLA, Billing & Wireless Neighbourhood \\
\hline \multirow{4}{*}{$\begin{array}{l}\text { Access Provision } \\
\text { Brokerage }\end{array}$} & Signal Strength & & $\begin{array}{ll}\text { Network, } & \text { Wireless } \\
\text { Neighbourhood } & \\
\end{array}$ \\
\hline & Time to Next Handover & & Network \\
\hline & $\begin{array}{l}\text { Connection options in the } \\
\text { Neighbourhood }\end{array}$ & All & $\begin{array}{l}\text { Network, Wireless } \\
\text { Neighbourhood. Environment }\end{array}$ \\
\hline & Incentives (to connect) & All & $\begin{array}{l}\text { Environment, } \quad \text { Wireless } \\
\text { Neighbourhood, Network }\end{array}$ \\
\hline \multirow{3}{*}{ Choice of Routes } & Trajectory & Security, Privacy, Rights & $\begin{array}{ll}\text { Service, } & \text { Environment, } \\
\text { Network } & \\
\end{array}$ \\
\hline & Incentives & All & $\begin{array}{l}\text { Environment, Wireless } \\
\text { Neighbourhood, Network }\end{array}$ \\
\hline & Signal Strength & & Wireless Neighbourhood \\
\hline \multirow{2}{*}{$\begin{array}{l}\text { LBS } \\
\text { (Location Based } \\
\text { Services) }\end{array}$} & Positioning & Privacy & Environment \\
\hline & Incentives (to shop) & & User \\
\hline
\end{tabular}

\subsection{The Pocket Switched Network}

In Pocket Switched Networking (PSN) by Hui et al. [3], there are three methods by which data can be transferred, namely neighbourhood connectivity to other local devices, infrastructure connectivity to the global Internet, and user mobility which can physically carry data from place to place. For the former two methods, the connectivity is subject to a number of characteristics, including those of bandwidth, latency, congestion, synchronicity (e.g. email or SMS are asynchronous, while ad-hoc WiFi is synchronous), the duration of the transfer opportunity (i.e. the time until the device moves out of range), and the monetary cost (usually only for infrastructure). For the latter method of user mobility, users acting as "data mules" can transfer significant amounts of data, and whilst users' movements cannot in general be controlled, they can be measured, and patterns in those movements can be exploited.

In PSNs like Haggle, which architecture appears in Figure 3, there are two broad classes of network demands which are known to be useful: (a) knownsender where one node needs to transfer data to a user defined destination. The destination may be another user (who may own many nodes), all users in a certain place, users with a certain role (e.g. "police"), etc. The key point is that, often, the destination is not a single node but is instead a set of nodes with some relationship, e.g. the set of nodes belonging to a message recipient. (b) known- recipient in which a device requires data of some sort, e.g. the current news. The source for this data can be any node which is reachable using any of the three connectivity types, including via infrastructure (e.g. a news webpage), neighbours (e.g. a recent cache of a news webpage) or mobility (e.g. the arrival of a mobile node carrying suitable data). In both classes described above, the endpoints of a network operation are no longer described by network-layer addresses, but are instead a set of desirable properties. As a result, general network operations no longer have single source and destination nodes.

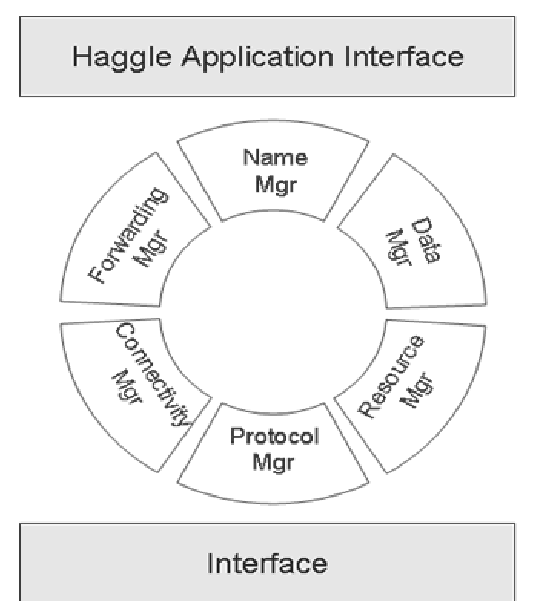

Figure 3. The Haggle architecture 
The three main motivations for a networking architecture in the PSN environment, in order of importance are:

- Allow applications to take advantage of all types of data transfer (neighbourhood, infrastructure, mobility) without having to specifically code for each circumstance.

- Allowing networking endpoints to be specified by user level naming schemes rather than nodespecific network addresses, thus each network operation can potentially involve many endpoints.

- Allowing limited resources to be used efficiently by mobile devices, taking into account user-level priorities for tasks.

The Table 2 shows some ways in which Haggle can use the SOHand information architecture.

Table 2. Some ways to combine sentient and opportunistic services at handovers

\begin{tabular}{|c|c|c|}
\hline Service & Parameters Involved & $\begin{array}{l}\text { Policies and Contexts Involved } \\
\text { (see Fig. 2) }\end{array}$ \\
\hline \multirow{6}{*}{$\begin{array}{l}\text { Deliver \& Collect Messages } \\
\text { (getting the exchange done) }\end{array}$} & Positioning and Timing & Environment \\
\hline & Discretion & Security, Privacy, Environment \\
\hline & Resources Availability & User device \\
\hline & Choice of Route & Environment \\
\hline & Weather Conditions & Environment \\
\hline & Incentives & All of them \\
\hline \multirow{5}{*}{$\begin{array}{l}\text { Announcements of Public } \\
\text { Services } \\
\text { (known-sender) }\end{array}$} & Positioning and Timing & Environment \\
\hline & Resources Availability & User Device \\
\hline & Wireless in the Neighbourhood & Network \\
\hline & Choice of Routes & Environment \\
\hline & Incentives & All of them \\
\hline \multirow{3}{*}{$\begin{array}{l}\text { Cache Sharing } \\
\text { (looking for packets to forward) }\end{array}$} & Positioning and timing & Environment \\
\hline & Resources Availability & User Device \\
\hline & Discretion & Security, Privacy, Environment \\
\hline \multirow{3}{*}{$\begin{array}{l}\text { HGA - Have a Glimpse Around } \\
\text { (looking for connection options) }\end{array}$} & Wireless in the neighbourhood & Network \\
\hline & Incentives & All of them \\
\hline & Positioning & Environment \\
\hline
\end{tabular}

\section{Conclusions and other important issues}

This paper deals with the problem of managing services in a multi-provider environment for NGN Next Generation Networks. The solution described has two main characteristics. First, it empowers the user with the overall control of their connectivity to the Internet, giving them the option to choose the provider which would suit them best at any moment. Second, it assumes that occasional, ad hoc communication with surrounding wireless-powered devices is possible and will be transparently managed by the system.

Due to regulations which, in certain countries such as the USA, prohibit the sending of unsolicited messages, passing the control of the roaming to the user might mean that Location Based Services (LBS) will become more feasible. This is because the users, by controlling their own context variables, could signal their desire to receive information about businesses in the area that they are visiting. With a network-centric way of management these services could be hard to implement without breaking the law.
On the other hand, the very same feature of controlling their user's personal context variables could be useful to disguise the user's presence, something which has proven useful in messaging applications such as Skype and MSN.

The ontological management has several advantages, the main one being the fact that it creates a common, semantically rich, vocabulary for the industry, which will be essential for the interoperation of the providers. With this semantics the search for services from different providers and its comparison in terms of quality will be eased. Additionally, the system helps the content designers to explore contextual information, such as that provided by Sentient Vehicles.

The implementation of the system, as a useful tool, depends on several conditions, from the clear need to create alternative architectures which will allow for a better reading of network information (away from the traditional layer-based models), all the way to the necessary vision of the telecommunication industry and governmental regulatory entities. It is hard to believe that the 
telecommunication industry would easily accept the paradigm change necessary to let the users to take control of handover choices as they roam. Eventually, the pressure of the content industry, notably the broadcasters, will lead regulatory agencies to signal the changes which are needed. However, in any case, the definition of a common, semantically rich set of vocabulary is a valuable tool for the entities involved in making services reliably available to mobile users.

\section{Acknowledgements}

We are grateful to CAPES, a Brazilian Education Ministry Agency and FAPESP, a Sao Paulo State Research Funding Agency, for the supporting for this work through the grants given to Edson D.S. Moreira and Renata M. P. Vanni

\section{References}

[1] AAA Resources on the web, Available: http://ch.tudelft.nl/ arthur/aaa/links.html

[2] S. Amit Kumar and B. J. David, Modeling mobility for vehicular ad-hoc networks. In Proceedings of the 1st ACM international workshop on Vehicular ad hoc networks Philadelphia, PA, USA: ACM Press, 2004.

[3] A. Chaintreau, P. Hui, J. Crowcroft, C. Diot, R. Gass, and J. Scott, Impact of Human Mobility on the Design of Opportunistic Forwarding Algorithms. In IEEE INFOCOM, Barcelona, Spain, 2006.

[4] H. Chen, F. Perich, T. Finin, and A. Joshi, SOUPA: Standard Ontology for Ubiquitous and Pervasive Applications. In First Annual International Conference on Mobile and Ubiquitous Systems: Networking and Services (MobiQuitous'04), 2004, pp. 258-267.

[5] M. A. Fernández, A. Gómez-Pérez, and N. Juristo, METHONTOLOGY: From Ontological Art towards Ontological Engineering. In AAAI Spring Symposium, Menlo Park, California, 1997, pp. 33-40.

[6] Google Mobile, Available: http://www.google.com/mobile/

[7] A. Hecker, H. Labiod, G. Pujolle, H. Afifi, A. Serhrouchni, and P. Urien, A New Access Control Solution for a Multi-Provider Wireless Environment. Telecommunication Systems, vol. 29, pp. 131-152, 2005.
[8] S. Jones, Toward an acceptable definition of service [service-oriented architecture]. Software, IEEE, vol. 22, pp. 87-93, May-Jun 20052005.

[9] L. W. Lacy, OWL: Representing Information Using the Web Ontology Language. Trafford, 2005.

[10] B. M. Michelson, Event-Driven Architecture Overview: Event-Driven SOA Is Just Part of the EDA Story, private communication, 2006.

[11] Mobile Information Device Profile, Available: http://java.sun.com/products/midp/

[12] Mobile Web Services, Available: http://www.microsoft.com/serviceproviders/resources/bizre smws.mspx

[13] I. G. Niemegeers and S. M. H. d. Groot, From Personal Area Networks to Personal Networks: A User Oriented Approach. Wireless Personal Communications, vol. 22, Number 2, pp. 175-186, 2002.

[14] Protègè, Available: http://protege.stanford.edu/

[15] Jonathan J. Davies, David N. Cottingham and Brian D. Jones, A Research Platform for Sentient Transportation. LNCS volume 4272, pp 226-229, 2006.

[16] Simple Object Access Protocol, Available: http://www.w3.org/TR/soap/

[17] P. Vidales, Seamless mobility in 4G systems. Computer Laboratory, University of Cambridge, 2005, p. 141. Ph.D. dissertation.

[18] P. Vidales, G. Mapp, F. Stajano, and J. Crowcroft, A Practical Approach for 4G Systems: Deployment of Overlay Networks. In First International Conference on Testbeds and Research Infrastructures for the DEvelopment of NeTworks and COMmunities (TRIDENTCOM'05), 2005, pp.:172 - 181.

[19] Web Services, Available: http://forum.nokia.com/main/resources/technologies/web_s ervices/index.html

[20] G.-Z. Yang, Body Sensor Networks. Springer, 2006

[21] David N. Cottingham, Wireless Coverage Mapping in Vehicular Contexts. Submitted to ACM CoNext, 2007.. 